Lexis Vol. XLI (2) 2017: 403-423

\title{
Subordinación locativa y modal en tehuelche o aonek’o Pa?jen. Aspectos sincrónicos y diacrónicos
}

\author{
Ana Fernández Garay \\ CONICET \\ Universidad Nacional de La Pampa
}

\section{RESUMEN}

El artículo analiza las subordinadas locativas y modales del tehuelche o aonek'o Pa?jen, lengua indígena de la Patagonia argentina hablada entre el río Santa Cruz y el Estrecho de Magallanes. Actualmente, se halla en un proceso avanzado de pérdida, aunque la comunidad tehuelche intenta revitalizarla. En el marco de la tipología funcional, se describen ambas cláusulas teniendo en cuenta el elemento que las introduce, su ubicación en la oración principal y el modo del núcleo predicativo. Asimismo, se consideran las similitudes existentes entre ambas. Por último, se explica el origen de los subordinantes y de las variantes que estos presentan. Para ello, se recurre a la comparación con el selknam, lengua perteneciente a la familia Chon, al igual que el tehuelche.

Palabras clave: tehuelche, subordinación adverbial, sincronía, diacronía

\section{AbSTRACT}

This article analyzes locative and manner clauses of the Tehuelche or Aonek'o Pa?jen, an indigenous language of the Argentinian Patagonia, spoken between the Santa Cruz river and the Strait of Magellan. At present, this language is facing an advanced process of extinction, despite 
Tehuelche community efforts to revitalize it. Locative and manner clauses are described according to a functional-typological frame, by taking into account the element that introduces them, their location in the sentence and the mood of the predicative core. In addition, the similarities found between both clauses are considered. Finally, the origin of the subordinators are explained by comparing them with those found in Selknam, a language that, like Tehuelche, belongs to the Chon family.

Keywords: Tehuelche, adverbial subordination, synchrony, diachrony

\section{O. Objetivo}

En este artículo nos ocuparemos de dos tipos de cláusulas adverbiales, las locativas y las modales. Describiremos sus subordinantes, el modo verbal que presentan y su ubicación en la oración compleja. Asimismo, intentaremos establecer el origen del subordinante que introduce ambas proposiciones: Paj, ${ }^{1}$ Pajk' y Pajk'er, y sus variantes. Nuestra hipótesis es que provienen del verbo transitivo del Grupo 1 - aj 'poner algo en algún lugar', del que derivan también las posposiciones concordantes - aj 'en', -ajk' 'hacia un punto' y -ajk'er 'desde un punto’. Para el estudio diacrónico, se considerará la existencia de un verbo del selknam, lengua hablada en Tierra del Fuego y actualmente extinta, del que derivan también elementos funcionales (posposiciones y subordinantes). Dicho verbo es cognado del verbo tehuelche mencionado.

\section{El tehuelche o aonek'o Pa?jen}

El tehuelche es una lengua prácticamente extinta, perteneciente a la familia Chon junto con el teushen, el selknam y el haush (Suárez 1988: 79-100). Esta se hablaba en la Patagonia argentina, entre el río Santa Cruz y el Estrecho de Magallanes. Se caracteriza por

\footnotetext{
1 La notación empleada es fonológica. Los fonemas del tehuelche son los

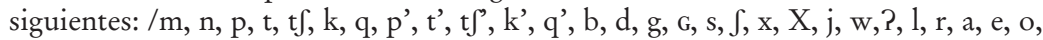
a:, e:, o:/.
} 
ser aglutinante, marcar el núcleo y poseer un orden básico SOV,² aunque el orden OVS es bastante frecuente, por lo cual presenta mayoritariamente posposiciones. Es una de las lenguas más documentadas de la Patagonia, después del mapuzungun, con el que ha estado en contacto y del que ha recibido influencias en su estructura fonético-fonológica, morfosintáctica y léxica.

Existe en aonek'o Pa?jen la oposición verbo-nombre, es decir, que hay una clase verbal que se usa exclusivamente como predicado o núcleo de enunciado, aunque también otras clases, como los sustantivos, demostrativos, indefinidos, personales, funcionales, ${ }^{3}$ cuantitativos y cuantificadores pueden predicativizarse al ser determinados por algunas categorías verbales. Debemos destacar que la posibilidad de calificar está dada por los verbos atributivos del Grupo 1 (véase más abajo) que se caracterizan por concordar en género con el participante único. Existe, además, la clase de los proposicionales, es decir, aquellos morfemas que pueden remplazar por sí mismos una proposición; ellos son k'om 'no' y orj 'si'. Los sustantivos se agrupan, según las influencias que ejercen sobre los significantes de los morfemas con los cuales se relacionan, en masculinos, femeninos y neutros. La elección de un sustantivo particular determina una serie de variaciones morfológicas en los miembros de las diferentes clases con los que concuerda: verbos, funcionales, categorías verbales, e incluso los mismos sustantivos cuando se combinan en la construcción posesiva (véase Fernández Garay 1998: 180 y ss.). Los sustantivos que presentan posesión inherente u obligatoria derivan de verbos transitivos del Grupo 1 que indexan necesariamente el objeto (véase más abajo). Los personales

\footnotetext{
2 Las abreviaturas utilizadas en este trabajo son las siguientes: A 'agente de oración transitiva', ADP 'adposición', CAU 'causativo', DIR 'direccional', DU 'dual', DUR 'durativo', EP ‘especificador del predicado', F 'femenino', M 'masculino', MED 'voz media', MI 'modo imperativo', MNR 'modo no real', MR 'modo real', N 'neutro', O 'objeto', PL 'plural', s 'sujeto', SM 'soporte de modalidades', TFI 'tiempo futuro de intención’, TFM ‘futuro mediato', TPL 'tiempo pasado lejano’, TPRCT 'tiempo reciente’, UA 'unidad asintática', v 'verbo', 1, 2, 3 'primera, segunda y tercera persona'.

3 Los funcionales son morfemas que sirven para indicar la función de otros elementos, como por ejemplo las posposiciones o preposiciones.
} 
se dividen en dependientes e independientes. Los dependientes son clíticos que generalmente se prefijan a verbos, sustantivos, adverbios, indefinidos, adposiciones, y también pueden sufijarse a verbos en modo imperativo.

Los verbos intransitivos y transitivos del tehuelche se dividen en dos grupos: los intransitivos del Grupo 1 concuerdan en género con el sujeto por medio de las formas $k-\sim ?-/ \varnothing$ - prefijadas al verbo: $k$ - concuerda con un participante único masculino o femenino; ?- / $\varnothing$-, con un participante único neutro (la forma $P$ - se convierte en $\varnothing$ - ante consonante). Desde el punto de vista semántico son atributivos. Véanse los siguientes ejemplos:

(1) $e-k-t \int^{\prime} e m a l e-f-k^{34}$

$1-\mathrm{M} / \mathrm{F}-$ estar.lastimado-EP-MR

'yo estoy lastimado'

(2) tf'am Ø-tf'emale-f- $k$ '

labio.N N-estar.lastimado-EP-MR

'el labio está lastimado'

(3)
P-ajq'e-f-k'-n e-Por
$\mathrm{N}$-ser.chata-EP-MR-N mi-nariz.N
'es chata mi nariz'

Los intransitivos del Grupo 2 no concuerdan en género con el participante único. Son verbos mayoritariamente de acción (4), aunque también pueden ser atributivos (5): ${ }^{5}$
(4) $t-\int-t-a \int$
tfotfao-S qawel-je
3-PL-3-entre bailar-EP Qawel-M
'entre ellos bailaba Qawel'

\footnotetext{
4 Los ejemplos cuyas fuentes no se indican provienen del corpus general registrado por Fernández Garay in situ.

5 Es posible que los índices genéricos que caracterizan este grupo de verbos intransitivos hayan desaparecido en algunos verbos atributivos por el estado terminal de la lengua.
} 

(5) gajaq'e-f-k'-e Pem gato
ser.cariñoso-EP-MR-M ese gato.M
'es cariñoso este gato'

Los verbos transitivos del Grupo 1 concuerdan con el objeto por medio de las formas $k-\sim$ ?- / Ø- "tercera persona paciente indeterminada" ( $k$-implica que $\mathrm{O}$ es masculino o femenino, y ?- / $\varnothing$-, que $\mathrm{O}$ es neutro). Ahora bien, hay una diferencia entre el índice genérico $k$ - ?- / Ø- de los verbos intransitivos del Grupo 1 y la "tercera persona paciente indeterminada" de los verbos transitivos, tal como la hemos llamado. En el primer caso, dichos prefijos solo indican concordancia de género con el sujeto, y no distinguen persona, pues el verbo puede aparecer determinado por la primera, segunda o tercera persona como se ve en el ejemplo (1). En el segundo, indican una tercera persona indeterminada en función de objeto, y conmutan con los otros personales dependientes, como se observa en los ejemplos que siguen:

(6) $e-k$-erno- $f-k$ '-e

1-3.M/F-dejar-EP-MR-M mi-marido.M

'yo (lo) dejé a mi marido'

(7) $e-m-e r n o-f-k$ '

1-2-dejar-EP-MR

'yo te dejo'

$\begin{array}{lll}\text { (8) kaj } \quad P-a X e-\int-k \text { '-n } & \text { wen ka:rken } \\ \text { capa.N } & \text { 3.N-pintar-EP-MR-F/N } & \text { esta mujer.F } \\ \text { 'una capa pinta esta mujer' } & & \end{array}$

En (6) y (7) - $m$ - conmuta con - $k$ - (“yo te” / “yo lo”). En (8) el índice genérico $?$ - concuerda con el paciente $k a j$ 'capa' que es neutro.

Los verbos transitivos del Grupo 2 no concuerdan en género con $\mathrm{O}$ cuando este se expresa por medio de una frase nominal: 
(9)
p'aXer $\quad \int \quad e-m a-k$ '
liebre ADP 1-matar-MR
'una liebre yo maté'

El verbo presenta las siguientes categorías flexivas: persona, género, número, tiempo, modo, especificador de predicado ${ }^{6} \mathrm{y}$ direccionales. Salvo en los verbos del Grupo 1 donde $\mathrm{O}$ está indexado en el verbo obligatoriamente a través de $k-\sim$ ?- / Ø- "tercera persona indeterminada en rol paciente”, o por medio de los personales dependientes, todas las demás categorías son facultativas, es decir, pueden no estar presentes en la oración declarativa. Los morfemas aspectuales, causativos y aplicativos se presentan como afijos derivativos.

La estructura sintáctica del tehuelche es nominativo-marcada (Dixon 1994: 63-67) y coexiste con un subsistema ergativo-absolutivo que se encontraba en proceso de retracción al momento de describir la lengua, en tanto que el primer sistema se iba imponiendo, tal como pudo comprobarse a partir de la comparación de textos de distintas épocas (Fernández Garay 2000: 173-174). En el sistema nominativo-marcado, la marca de $\mathrm{S}$ de la oración intransitiva y de A de la transitiva es la adposición $\int \sim n \sim r^{7}$ (véase en (9) la adposición $\int$ que marca el agente $e^{-}$) en tanto que, en la transitiva, la frase nominal en función de objeto concuerda con el verbo en género y persona si este pertenece al Grupo 1, pero, si el verbo pertenece al Grupo 2, solo presenta facultativamente concordancia de persona. El subsistema ergativo-absolutivo se manifiesta en los verbos del Grupo 1 que concuerdan con S/O en tanto que A puede mostrar la marca adpositiva mencionada, dado que ambos sistemas coexisten en la actualidad (véase Fernández Garay 2007: 114-125).

\footnotetext{
6 El especificador del predicado es un morfema que con su presencia indica la existencia de un verbo o de un morfema de otra clase que se ha convertido en predicado. 7 marca el agente cuando el verbo presenta modo real, se emplea la variante $n$ cuando el verbo está determinado por el modo no-real, y $r$ marca el agente en la interrogación.
} 
La lengua exhibe preferentemente posposiciones, y algunas adposiciones, que funcionan a veces como posposiciones y en otros casos como preposiciones, posiblemente por el largo contacto con el mapudungun primero y el español después. Se observan casos de incorporación nominal aunque esta es muy incipiente y adquirida probablemente por contacto con el mapudungun. Incluye verbos negativos, entre ellos, $k$ 'om 'faltar', que funciona como predicado

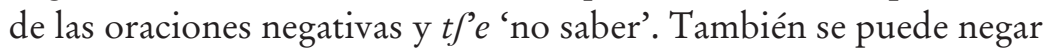
por medio del adverbio ken 'no' y del sufijo prohibitivo ?e:w.

\section{Aspectos teóricos}

Según Thompson, Longacre y Hwang (2007: 237) la relación entre las cláusulas principal y subordinada es claramente un continuum, uno de cuyos extremos es una cláusula gramaticalmente dependiente de otra o de algún elemento de dicha cláusula. Así, las argumentales - que funcionan como frases nominales dependientes de un verbo-y las relativas - que funcionan como modificadoras de un núcleo nominal o adverbial- serían las más "incrustadas" (embedded), en tanto que las adverbiales — que determinan a la FV o a la oración completa-, si bien son vistas como hipotácticas o subordinadas a una principal, pues de algún modo dependen de ellas, sin embargo, son las menos subordinadas de las tres.

Según Thompson et al. (2007), hay tres estrategias para subordinar cláusulas: i. morfemas subordinantes, ii. formas especiales del verbo y iii. orden de palabras. En cuanto a i., hay dos formas de subordinantes: morfemas gramaticales sin contenido léxico (to en inglés) y morfemas gramaticales que presentan contenido léxico (cuando, donde, etc.). Estos elementos subordinantes - conjunciones y adposiciones- pueden iniciar la cláusula en una lengua con núcleo inicial (VOS) o colocarse al final de la cláusula en una lengua con núcleo final (SOV). En lo que refiere a ii., las formas especiales de los verbos son aquellas que no son usadas en las cláusulas independientes. Pueden ser formas no finitas, como también otro tipo de formas verbales. Con respecto a iii., se dice que hay 
lenguas que en las cláusulas subordinadas presentan un ordenamiento distinto del que se observa en la cláusula principal. Otro aspecto a considerar sobre las subordinadas adverbiales es su posición en la oración. En algunas lenguas suelen preceder a la principal, en tanto que en otras se colocan por detrás de las mismas. Se destaca que muchas veces las lenguas carecen de subordinadas para expresar ciertos contenidos adverbiales, y en su lugar se emplean cláusulas yuxtapuestas o construcciones seriales verbales.

Se han reportado doce tipos de subordinadas adverbiales, tres de ellas pueden ser sustituidas por un adverbio. Ellas son las temporales, espaciales y modales. Las que no pueden ser sustituidas por un adverbio son las finales, causales, concesivas, condicionales, simultáneas (mientras), aditivas (además de), absolutivas (con nominalizaciones) circunstanciales (por medio de, sin) y sustitutivas (en lugar de).

Con respecto a las tres primeras, dicen Thompson et al. (2007: 244), que presentan un rasgo tipológico interesante y es que tienden a tomar la forma y compartir las propiedades con las cláusulas relativas. Y esto ocurre porque las cláusulas temporales, espaciales y modales establecen que la relación entre el tiempo, el lugar o el modo del evento de la cláusula principal y el de la subordinada es el mismo. Y es por esto que, generalmente, comparten propiedades con las cláusulas relativas:

I'll meet you where the statue used to be.

I'll meet you at the place at which the statue used to be.

Así, el pronombre relativo at which indica la locación en la cláusula relativa y la FN at the place at which... indica la locación en la principal, o sea, remiten a un mismo espacio.

Tomaremos también en cuenta a Cristofaro (2005), para quien el criterio morfosintáctico no es lo fundamental para su planteo, ya que la subordinación debe ser definida en términos funcionales. Así, la subordinación es vista como un modo particular de construir una relación cognitiva entre dos eventos, de manera tal que uno de ellos, el dependiente, carece de perfil autónomo y es 
construido en la perspectiva del otro llamado principal, es decir, la subordinación concierne a las relaciones cognitivas entre eventos y es independiente de la manera en que la unión de las cláusulas se realiza translingüísticamente.

$\mathrm{Su}$ perspectiva teórica se enmarca dentro del funcionalismo tipológico. Su carácter distintivo se encuentra en el énfasis puesto en la comparación translingüística y el rol crucial que juegan los factores funcionales, más que estructurales, en todos los niveles de análisis. La función lingüística motiva la estructura lingüística pues la forma de la última, aunque no derivable de la primera, la refleja hasta cierto punto. Las dos motivaciones funcionales más importantes son la iconicidad y la economía. La primera es la tendencia a modelar la estructura lingüística según la perspectiva impuesta por la experiencia conceptual del hablante. Esto conforma una estructura lingüística altamente transparente. La otra es la economía que implica la presión hacia el mínimo esfuerzo y la máxima simplificación en la expresión.

Propone una definición funcional de la subordinación, según la cual esta implica una situación que establece una asimetría cognitiva entre dos estados de situación (state of affairs-SoAs-), de modo tal que el perfil de uno de los dos, el estado de situación principal, invalida al otro, el dependiente. Esto equivale a decir que el dependiente está pragmáticamente no aseverado, mientras el principal sí lo está. Esta suposición de asimetría es independiente de las propiedades estructurales de cualquier tipo de cláusula y permite identificar los estados de situación dependientes translingüísticamente.

Resumiendo, la subordinación es definida como una relación conceptual/pragmática asimétrica entre dos estados de situación. Tiene en cuenta dos parámetros de análisis: la forma del verbo y la codificación de los participantes. Para ello se comparan los que aparecen en cláusulas declarativas independientes con los que aparecen en las dependientes. Esto es una estrategia funcional, pues define las formas verbales en base a los tipos de cláusulas que pueden expresar. En esto se fundamenta para plantear las dos estrategias que ella denomina el equilibrio y el descenso de rango. 
En la estrategia del equilibrio los dos tipos de cláusulas (dependiente e independiente) presentan formas verbales estructuralmente equivalentes. En la estrategia de la degradación, una de las cláusulas puede expresarse por medio de un verbo que no puede ser usado en cláusulas independientes (2005: 54-55).

\section{Cláusulas adverbiales espaciales en tehuelche}

Las cláusulas adverbiales espaciales o locativas se introducen por medio del subordinante $P a j \sim a j$ 'donde' y Pajk'er ajk'er 'desde':

(10) jomno kenof $m$-f-m-a:jxen-f-kPo <m-f-Paj pe-m> cualquier momento 2-PL-2-visitar-EP-TFI 2-PL-donde asentarse-MNR 'En cualquier momento iré a visitarlos donde ustedes viven'.

(11) e?wn-f-k'-en <j-ajk'er Pa-m-ote> lejos-EP-MR-F/N 1-de donde SM-MNR-DIR 'Es lejos de donde vengo'.

Se observa que en nuestro corpus, el subordinante puede presentar las formas indicadas más arriba. Sin embargo, en textos anteriores (Fernández Garay y Hernández 2006; ${ }^{8}$ Fernández Garay $2009^{9}$ ) encontramos otras formas de este subordinante:

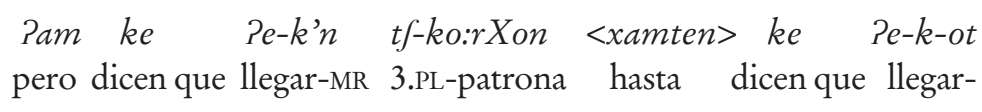
$<$ kaj Pem-je ke $t$-Pe- $s$ donde ese-M dicen que 3-llegar-EP

\footnotetext{
Este volumen contiene textos recogidos por Jorge Suárez entre 1966 y 1968.

9 Este libro reúne cinco textos registrados por Lehmann Nitsche en 1905, cuando un grupo de tehuelches regresaba de una Exposición Internacional realizada en Saint Louis, Missouri, Norteamérica (Fernández Garay 2009: 17). La primera notación de la frase es la realizada por Lehmann Nitsche, tomando como base la ortografía alemana; la segunda es fonológica (ver nota 3). La traducción entre comillas es la de Lehmann Nitsche, y la que se encuentra entre corchetes es de Fernández Garay, al igual que el análisis morfológico.
} 
'Pero dicen que llegó la patrona de ellas, que llegó hasta donde ese había llegado'.

(Fernández Garay y Hernández 2006: 66-67)

(13) áan áshkn tám áishpn t’änun käu üksh ush uänolpsh aik nush á an en ush ään mo\|

Pa:n Pafk'n ta: mPajfpn t'e:non kewek $\int$ ofwe:nolf<Pajk' n ofPa Pane nof Pe:nm?o>

Pa:n Pa-f-k'-n ta: mPaj- $\int p-n \quad$ t'e:non k-ewek $\int$ mucho SM-EP-MR-N 3 hacer ruido-DUR-F/N noche.F M/F-cada ADP

o-f-we:nol-f <Pajk' $n \quad$ o-f-Pa Pane $n$ o-f-Pe:n-m-Po> 1-PL-pasear-EP adonde ADP 1-PL-UA UA ADP 1-PL-ir-MNR-DIR 'Todas las noches había mucho ruido y paseábamos adonde nosotros fuimos'

[Hacían mucho ruido, y cada noche paseábamos adonde habíamos ido]

(Fernández Garay 2009: 115-116)

En los ejemplos (12) y (13) encontramos las formas kaj y Pajk' respectivamente, las que no fueron documentadas durante nuestros trabajos de campo. Ahora bien, de los distintos ejemplos podemos observar lo siguiente:

1. Los subordinantes se posponen a un clítico personal en (10) y (11), y aparecen así en segunda posición dentro de la cláusula subordinada.

2. Los subordinantes se ubican al comienzo de la subordinada en (12) y (13).

3. Los verbos de las cláusulas espaciales están en modo no-real (10), (11) y (13) o carecen de modo (12), ya que esta categoría flexiva del verbo no es obligatoria.

4. En los cuatro ejemplos no hay un elemento adverbial que sirva de antecedente a la cláusula subordinada.

Veamos ahora los siguientes ejemplos: 
(14) kenk t-we:-m-n m-f-Pajk'e-n-ne Pemne <m-f-Paj pe-m> cómo 3-ser-MNR-N 2-PL-vivir-FNF-N allá 2-PL-donde asentarse-MNR ‘Cómo es su vida allá, donde viven ustedes?'

(15) Pa: j-e:wkale-f-k-Po ke Pe- $\int$ welom ke UA 1-ganar-EP-MR-DIR dicenque decir-EP todo dicenque

Sawxe-f wene $<t$-aj $t$ - $\int$ o: $r-m e-t \int>$ sangrar-EP acá 3-donde 3picotear-MNR-PL

" "Ah, me ganaron», dicen que dijo. Dicen que estaba todo ensangrentado ahí donde lo picotearon’.

En los ejemplos (14) y (15) notamos un elemento adverbial que precede a la cláusula subordinada, que por esto mismo ha dejado de ser una subordinada adverbial para convertirse en una relativa, tal como proponen Thompson et al. (2007: 244). En este sentido, adverbiales y relativas espaciales solo se distinguen por el adverbio de lugar que precede a la cláusula subordinada. Además, vemos nuevamente que en estos ejemplos el subordinante se ubica en segunda posición, siendo la primera ocupada por un clítico personal, como ocurre en los ejemplos (10) y (11).

Debemos destacar que en todos los casos, las cláusulas adverbiales espaciales se ubican al final de la oración, al igual que las relativas con valor locativo.

\section{Cláusulas adverbiales modales en tehuelche}

Las cláusulas adverbiales modales son introducidas por medio del subordinante Pajk' ajk' 'como':

...paj Pem-tf Pajk'e-f-m <f o-f-Pajk' mtf'enje-f-nk'er naf> pues ese-PL vivir-EP-MNR ADP 1-PL-como conversar-EP-TRCT ayer '...porque esos vivían como nosotros conversábamos ayer'. (17) Pemaj o-f-ka:w Paw wa:le-f ma? <n o-k-Pajk' Pere:-f-m o-f-wa: > pues 1-PL-toldo después andar-EP ahora ADP 1-DU-comoandar-EP-MNR 1-PL-1 
'Pues ahora andamos por sitios donde hubo tolderías, como andamos nosotras dos'.

En (18) aparece un subordinante distinto al que encontramos en nuestro corpus: Paj -aj:

(18) xelo kajokon wen ma?k'o wenk'o re:je $\langle n$ o-f-Paj Pa-m> porque otro este nuevo esta andanza ADP 1-PL-como SM-MNR 'Porque este modo moderno de andar es diferente a como era el nuestro'.

(Fernández Garay y Hernández 2006: 193)

En estos ejemplos, observamos lo siguiente:

1. Los subordinantes se ubican en segunda posición en la cláusula subordinada, siguiendo a un clítico personal en (16), (17) y (18).

2. Los verbos de las cláusulas modales están en modo no-real en (17) y (18) o carecen de modo (16).

3. En los tres ejemplos no hay un elemento adverbial que sirva de antecedente a la cláusula subordinada.

En los ejemplos que siguen, vemos las subordinadas funcionando como relativas modales, con el antecedente adverbial newr newrk' 'asî':

$k$-Pokom-tf // newrt-we:- $k^{\prime} / /<t-a j \quad$ Pema?-m-n wenaj> REFL-seguir-PL así 3-ser-MR 3-como pasar-MNR-F/N acá 'Se siguieron (uno al otro); así fue la cosa, como pasó acá'

(20) Pafo xarwke-k'-e newrk' $P e<n$ o-k-Pajk' $2 a-m$ Pa t'enje- $\int>$ solo dos-MR-M así UA ADP 1-DU-como SM-MNR UA conversar-EP 'Así como conversamos nosotros, somos solo dos, ¿eh?'

Así pues, las cláusulas adverbiales espaciales y modales se caracterizan por estar introducidas por subordinantes cuya procedencia veremos a continuación. Asimismo, otra característica es la ubicación por detrás de la oración principal como elemento final al igual 
que las relativas espaciales y modales, con el agregado de que estas exigen un antecedente adverbial. Por último, presentan el mismo modo no-real. Lo único que parece diferenciarlas, además del adverbio que precede a la relativa, es que las adverbiales presentan un menor grado de subordinación que el que presentan las cláusulas de relativo, pues estas, al depender de un adverbio, se hallan incrustadas en la cláusula principal. Podemos afirmar que en el continuum del que hablan Thompson et al. (2007), las adverbiales aquí analizadas se encontrarían dentro de las subordinadas, pero alejándose del extremo de las denominadas “incrustadas". El subordinante generalmente se ubica en segunda posición dentro de la cláusula, por detrás de un clítico personal. Es menos frecuente su ubicación al comienzo de la subordinada.

\section{Origen de los subordinantes}

Resumiendo, podemos decir que las cláusulas adverbiales locativas y modales emplean el mismo subordinante $P a j \sim a j$ y difieren en el uso de uno de los lexemas derivados de este, ya que las locativas emplean, entre otros, el funcional Pajk'er ajk'er, en tanto que las modales no fueron documentadas hasta el momento introducidas por el mismo. Ahora bien, estos subordinantes derivan de una posposición concordante de esta lengua: - aj 'en', que posee un sentido general de estar en un lugar determinado ${ }^{10}$ (Fernández Garay 1998: 297 y ss.). Las posposiciones concordantes se caracterizan por variar sus significantes según el género del sustantivo al que se posponen, es decir, prefijan $k$ - si dicho sustantivo es masculino o femenino, y ?- / Ø- si el mismo es neutro, tal como se observa también en los verbos del Grupo 1, aquellos que concuerdan en género con el sujeto cuando son intransitivos y con el objeto cuando son transitivos. También pueden ser invariables si sus significantes no modifican su forma con respecto al género, aunque no nos ocuparemos acá de

10 Debemos destacar que Heine y Kuteva proponen la posposición locativa como origen o fuente de subordinantes (2002: 205). 
ellas porque no están relacionadas con el tema que nos ocupa. A su vez, las posposiciones concordantes pueden ser monomorfemáticas si están constituidas por una unidad significativa, o derivadas si a un morfema de base se agregan sufijos derivativos. A continuación, se presenta la nómina de las posposiciones:

Las posposiciones concordantes monomorfemáticas son las siguientes:

\begin{tabular}{|c|c|c|c|}
\hline$-a m \int$ & 'bajo, debajo de' & -eno & 'junto a' \\
\hline$-a \int$ & 'en, dentro de' & -eren & 'a causa de' \\
\hline$-a j$ & 'en, durante'11 & -e:wek & 'cada' \\
\hline -ajken & 'detrás de’ & $-m a j$ & 'a causa de' \\
\hline$-a w$ & 'después de' & -naon & 'para' \\
\hline$-a w r$ & 'sobre, encima de' & -or & 'alrededor' \\
\hline -a:nte & 'por, a causa de' & $-\operatorname{or} x$ & 'delante de' \\
\hline$a x$ & 'por' & $-x o \int$ & 'a la orilla de' \\
\hline
\end{tabular}

Para mostrar la variación, según el género de estas posposiciones monomorfemáticas, observemos los ejemplos (21) y (22):

$$
\begin{aligned}
e-\text {-wat'e-f le? } & k \text {-a } \\
\text { 1-caer-EP agua.M } & \mathrm{M} / \mathrm{F}-\mathrm{en} \\
\text { 'Caí en el agua' } &
\end{aligned}
$$

(22) ka:w P-af $\int \frac{o-f-p e-k}{}$ '
toldo.N N-en ADP 1 -PL-estar-MR
'Estamos en el toldo'

Vemos, además, que algunas de las que forman la clase de las posposiciones provienen de verbos, como se muestra a continuación:

11 Existe un tipo de subordinada adverbial temporal que es introducida por las posposiciones concordantes -aj, -ajk', -ajk'er y -orf, que se ubican por detrás de las formas no finitas del verbo (infinitivos) y que introducen una subordinada temporal más “degradada”, al decir de Cristofaro (2005), situación que no se documentó para las locativas y modales (véase Fernández Garay 2010). 


$$
\begin{array}{ll}
\text {-afe? 'entrar' } & >-a \text { 'en, dentro de' } \\
\text {-awre 'montar, tocar' }>\text {-awr 'sobre' } \\
\text {-enwe 'acompañar' }>- \text { eno 'junto a' } \\
\text {-orx 'rodear' } & >- \text { or 'alrededor' } \\
\text {-aj 'poner en' } & >-a j \text { 'en' }
\end{array}
$$

\begin{tabular}{|c|c|c|}
\hline$-a j k^{\prime}$ & 'hacia un punto' & 'desde un punto' \\
\hline$-a \int k^{\prime}$ & 'hacia adentro' & 'desde adentro' \\
\hline -awrk' & 'hacia encima de' & 'desde encima de' \\
\hline -enek’ & 'hacia junto a' & -enek'er 'desde frente a' \\
\hline -ewk' & 'hacia frente a' & $\begin{array}{l}\text {-ewk'er 'desde frente a' } \\
\text {-enok'er 'desde junto a' } \\
\text {-t'e:k'er 'desde atrás' }\end{array}$ \\
\hline
\end{tabular}

Las posposiciones concordantes derivadas agregan un morfema derivativo - $k$ ' hacia' y $-k$ 'er 'desde'. Fueron documentadas las siguientes:

Volviendo a la posposición que nos interesa, hemos visto que coexiste en sincronía con el verbo del Grupo 1 - aj 'poner algo en algún lugar', del cual deriva por gramaticalización. Este proceso continuó su avance, pasando a convertir la posposición en un afijo derivativo que encontramos sufijado al indefinido ken 'cuál, algo, cosa' dando lugar a kenaj 'dónde’. Asimismo, se sufija a los demostrativos, dando lugar a los deícticos adverbiales locativos: wen 'este' $>$ wenaj 'acá', Pem 'ese' > Pemaj 'alli', mer 'ese' > meraj 'allí, mon 'aquel' > monaj 'allá'.

Por otro lado, la posposición -aj es determinada por los sufijos $-k$ ' y -k'er. Desconocemos el origen de estos derivativos, pero es probable que ambos sean gramaticalizaciones de verbos de movimiento, dado el sentido alativo del primero y ablativo del segundo. Ambos se sufijan al adverbio kenaj 'dónde' dando lugar a los adverbios kenajk' 'adónde' y kenajk'er 'de dónde'. Otros adverbios determinados por estos derivativos son wenaj 'acá', que generan los adverbios wenajk' 'hacia acá' y wenajk'er 'desde acá'. Un adverbio 
que nos interesa es newr 'así', pues precisamente presenta un valor modal. El agregado del sufijo alativo genera un adverbio derivativo newrk', cuyo significado no presenta cambio alguno, lo que nos hace pensar que la gramaticalización con su proceso de desemantización lo ha llevado a adquirir un sentido más abstracto y metafórico que hoy no es posible determinar.

Ahora bien, en las cláusulas adverbiales locativas y modales vimos que, en general, se emplean las formas aj, ajk' y ajk'er y aquellas que prefijan la glotal Paj, Pajk’ y Pajk'er. La pérdida de la glotal en tehuelche es un fenómeno común debido al contacto con el español y a la situación de extinción de la lengua, sobre todo en los corpora registrados durante la segunda mitad del siglo XX. Los prefijos concordantes $k$ - y $P$-, que se encuentran en los verbos del Grupo 1 y en las posposiciones concordantes, se excluyen mutuamente con los personales ka:w Paj 'en el toldo (neutro)', k'o:n kaJ 'dentro el río (masc.)', $j$-awr 'sobre mí' (siendo $j$ el clítico de primera persona). Sin embargo, en las cláusulas locativas y modales hemos podido observar que, si bien en algunos casos el subordinante carece de glotal inicial al seguir a los personales - como vemos en (11) y (15)—, en los demás casos siempre aparece la glotal antepuesta al morfema aj. Evidentemente, no funcionan como una posposición, sino que han lexicalizado la glotal, con lo cual se convierten en un subordinante que mantiene aún el sentido locativo en las espaciales y un valor semántico más abstracto de manera en las modales. Solo encontramos una ocurrencia, el ejemplo (12), en la que aparece $k$ - antepuesta a dicho morfema. Esto puede explicarse por el desgaste y variación existente en una lengua que se halla en proceso de extinción.

Podemos afirmar, a partir de lo expuesto, que los subordinantes provienen de la posposición - aj, la que a su vez se origina en el verbo transitivo del Grupo 1 - aj que significa 'poner algo (en algún lugar)', verbo que toma $k$ - si el objeto es masculino/femenino, o ?si es neutro, como se ejemplifica en (23): 
(23)
Pajk'om k-ams jatf P-aj-f-k'
olla.m M/F-debajo de brasas.N N-poner-EP-MR
'Puso brasas debajo de la olla'

Ahora bien, Givón (1996: 379-384) presenta el posible desarrollo de posposiciones locativas estáticas a partir de verbos, cuando en general, como él mismo dice, estos suelen provenir de sustantivos o frases nominales (véase Heine y Kuteva 2002: 332). Comprueba su aserción con el ute, lengua Yutoazteca hablada en Colorado, Estados Unidos, donde claramente observa la gramaticalización de verbos que dan origen a posposiciones locativas de carácter estático.

Por otro lado, en selknam existen también verbos prefijables, como los llama Najlis (1973: 41), que son los que toman el prefijo $k$ que concuerda con el sujeto masculino o femenino si son intransitivos y con el objeto si son transitivos. El prefijo $b$-, en cambio, concuerda con sustantivos neutros en los mismos casos. Son, pues, similares a los del Grupo 1 del tehuelche. A su vez, en selknam existen alrededor de setenta posposiciones, como nos dice Najlis (1973: 51), que ella divide en prefijables (concordantes) y no prefijables (invariables). Entre ellas, -àj 'respecto de', que daría lugar a las siguientes formas, siguiendo el patrón de concordancia de los verbos prefijables:

(24) Pref. sorèn $k$-ăj ${ }^{12}$ 'respecto de la bolsa (F)'

(Najlis 1973: 51) kawj $b$-àj 'respecto de la casa (N)'

(Najlis 1973: 51)

También, en selknam existen varias conjunciones, una de ellas es hàj, que según la autora es la más frecuente y de significado más amplio: “temporal, espacial, causal, condicional” (1973: 50). Ofrece un ejemplo reducido de esta:
xej bàj-n t'elqn
viene cuando-F niña
'cuando viene la niña'

\footnotetext{
12 Los fonemas del selknam, según Najlis, son los siguientes: /p, t, c, k, q, p', t', k', q', s, ş, f, x, h, l, r, j, w, ?, m, n, i, e, è, u, o, ò, a, ̀̀/.
} 
Se observa que la conjunción subordinante se ubica por detrás del verbo y precediendo al sustantivo. Los valores suelen ser "cuando, porque, donde" (Najlis 1975: 41).

Evidentemente, el subordinante del tehuelche y el del selknam son cognados. En tehuelche, el subordinante y sus variantes generalmente prefijan ?-, y, en selknam, $b$-. Según Viegas (2005: 48), *? $\mathrm{y} * h$ - ambos fonemas del protochon - tienden a confundirse en tehuelche como ? o $\varnothing$, y en selknam, como ? o h. Además, estos subordinantes están directamente relacionados con las posposiciones concordantes y, en ellas, tienen su origen. Por otro lado, en selknam también existe un verbo transitivo prefijable a?j 'colocar (diciendo dónde)' (Najlis 1975: 66), que sería cognado del verbo tehuelche - aj. Si tenemos en cuenta los procesos de gramaticalización - que implican desemantización o pérdida de contenido signficativo; extensión o generalización de contextos, o uso en nuevos contextos; descategorización, o pérdida de propiedades morfosintácticas características de un ítem léxico que pertenece a un rango superior y pasa a uno de rango inferior y erosión o pérdida de sustancia fonética (véase Heine y Kuteva 2002: 2)—, podemos considerar que los subordinantes en ambas lenguas son gramaticalizaciones de verbos. Evidentemente, el paso de verbos a posposiciones, si bien no es algo corriente en lenguas del mundo, es un proceso posible (Givón 1996: 95-96). Para ello, es necesario un contexto específico para que ocurra el proceso de reinterpretación del ítem léxico. Aparentemente, el verbo 'poner' o 'colocar en cierto lugar', existente en el protochon, dio lugar ya en la protolengua a las posposiciones concordantes locativas y, posteriormente, a los subordinantes, cuyos valores semánticos son más abstractos aún, con lo cual generó los morfemas para introducir cláusulas locativas y modales.

\section{Conclusiones}

El análisis de las cláusulas adverbiales locativas y modales muestra similitud estructural además del empleo de los mismos subordinantes para introducirlas. Esto manifiesta claramente una relación 
semántico-funcional entre ambas, es decir, entre locación/espacio y manera/modo. En cuanto al origen de los subordinantes, es bastante claro que ya en el protochon existía un verbo 'poner, colocar (en algún lugar)' que habría dado origen a posposiciones, y estas a subordinantes con la función de introducir cláusulas locativas o modales. Este elemento quedó lexicalizado después de un período de variación posible. De ahí la aparición de kaj en lugar de Paj en alguno de los ejemplos presentados. Por último, este proceso se dio ya en la protolengua, es decir, antes de que el tehuelche y el selknam se separaran, pues es posible advertir los mismos subordinantes en ambas lenguas.

\section{Referencias bibliográficas}

Cristofaro, Sonia

2005 Subordination. Oxford: Oxford University Press. https://doi. org/10.1093/acprof:oso/9780199282005.001.0001

Dixon, R. M.W.

1994 Ergativity. Cambridge: Cambridge University Press.

FERnÁndez Garay, Ana

1997 Testimonios de los últimos tebuelches. Textos originales con traducción y notas lingüístico-etnográficas. Buenos Aires: Archivo de Lenguas Indoamericanas, Colección Nuestra América, Instituto de Lingüística, Facultad de Filosofía y Letras, Universidad de Buenos Aires.

1998 El tebuelche. Descripción de una lengua en vías de extinción. Anejo No 15. Estudios Filológicos. Valdivia: Universidad Austral de Chile.

2000 "Consecuencias lingüísticas de la situación de desgaste del tehuelche". En Memorias del V Encuentro Internacional de Lingüística en el Noroeste. Tomo II. Sonora: Unison, 165-177.

2007 "Coexistencia de dos sistemas sintácticos en tehuelche". International Journal of American Linguistics. 73, 1, 114-125. https://doi.org/10.1086/518337 
2009 Los textos tebuelches de Robert Lebmann-Nitsche (1905). München: Lincom Europa, Languages of the World / Text collections.

2010 "Las cláusulas temporales en tehuelche o aonek'o Pa?jen”. LIAMES. Linguas indígenas americanas. 10, 37-48.

Fernández Garay, Ana y Graciela Hernández

2006 Textos tebuelches (aonek'o Paijen). Homenaje a Jorge Suárez. München: Lincom Europa.

Givón, Talmy

1996 "La gramaticalización de verbos a posposiciones en ute”. En Memorias del III Encuentro de Lingüística en el Noroeste. Tomo I: Lenguas Indígenas. Vol. 2. Sonora: Unison, 359-404.

Heine, Bernd y Tania Kuteva

2002 Word Lexicon of grammaticalization. Cambridge: Cambridge University Press. https://doi.org/10.1017/cbo9780511613463

NajLis, Elena

1973 Lengua Selknam. Buenos Aires: Universidad del Salvador, Instituto de Filología y Lingüística.

1975 Diccionario Selknam. Buenos Aires: Universidad del Salvador, Instituto de Filología y Lingüística.

SuÁREZ, Jorge

1988 "Clasificación interna de la familia lingüística Chon”. En Estudios sobre lenguas indígenas americanas. Ed., María Beatriz Fontanella de Weinberg. Bahía Blanca: Universidad Nacional del Sur, Departamento de Humanidades, 79-100.

Thompson, Sandra, Robert Longacre y Shin J. Hwang

2007 "Adverbial clauses". En Language Typology and Syntactic Description. Vol. II. 2nd Edition. Ed., Timothy Shopen. Cambridge: Cambridge University Press, 237-300.

Viegas Barros, José Pedro

2005 Voces en el viento. Raíces lingüisticas de la Patagonia. Buenos Aires: Editorial Mondragón. 